\title{
EFFECT OF HEAT TREATMENT ON THE EXPANSIVITY OF A PYREX GLASS
}

\author{
By J. B. Saunders and A. Q. Tool
}

\section{ABSTRACT}

Heating a pyrex glass at different temperatures between $450^{\circ}$ and $750^{\circ} \mathrm{C}$. caused its mean linear expansivity between $20^{\circ}$ and $200^{\circ} \mathrm{C}$. to change more than 10 percent. This expansivity in the case of the original untreated specimen was approximately $0.0341 \times 10^{-4}$ and after treatment at temperatures near $750^{\circ} \mathrm{C}$. it increased to $0.0345 \times 10^{-4}$, while treatments at $450^{\circ} \mathrm{C}$. reduced it to $0.0307 \times 10^{-4}$. After any such changes the original expansivity could be restored by a heat treatment near $650^{\circ} \mathrm{C}$. Treatments between $650^{\circ}$ and $450^{\circ} \mathrm{C}$. appeared to reduce the expansivity rather steadily as the treating temperature was lowered.

These results indicate that there is some advantage in annealing this glass at the lowest practical annealing temperature since any reduction in the expansivity should increase the resistance of the glass to heat shock.

In a previous paper ${ }^{1}$ it was pointed out that in some cases the heat treating of glass in the annealing range noticeably affects the magnitude of the expansivity. In addition to this, very little is known concerning such expansivity changes except that they are seldom large. Without more definite data regarding their actual magnitude it would also seem possible, however, that under certain conditions they may have an appreciable effect on the serviceability of glass. Moreover, unless their magnitude is better known it may be suspected that these changes are a controlling factor in producing the dimensional changes which are usually very easy to detect if the dimensions of the glass sample are measured under standard conditions both before and after it receives heat treatment in the annealing range.

\section{CONTENTS}

I. Introduction

II. Specimens tested and apparatus used

III. Heat treatments of samples

IV. Methods of observation

V. Results

VI. General discussion

VII. Summary

802

802

803

805

809

\section{INTRODUCTION}

From the standpoint of what may be attained in the way of dimensional changes under the usual conditions of production, it is ordinarily not feasible to alter the density of most glasses more than 2 or 3 percent by varying the heat treatment; and in view of this it may be thought that any changes which can be produced in the expansivity by the same cause and under the same conditions would never greatly exceed such a limit. In the production of most glassware an expansivity change no greater than this would generally be considered as relatively unimportant. Practical considerations soon show, however, that definite data on this effect of heat treatment are required because of the possibility that the changes in the expansivity may

1 Tool, Lloyd, and Merritt, J. Am. Ceramic Soc., vol. 13, p. 653, 1930. 
materially exceed 3 percent for some glasses and also because even smaller changes can under certain special conditions assume a real importance. For example, relatively small changes of this sort, if they are not uniform throughout a piece of ware, may cause a differential expansion that can easily be detrimental whenever such ware is heated or cooled. Especially in articles designed for precision measurements, nonuniformities of this character may often be the source of very troublesome distortions; and such distortion may occur even when the differential expansions developed during normal temperature changes are so small that the accompanying stresses fall very far below the breaking limit. Presumably such conditions of nonuniformity are more likely to appear in a ware consisting of several parts fused together, although they may also be present in any article fabricated under conditions which preclude a thoroughly uniform and sufficient annealing treatment.

If certain glasses are found for which the relative changes produced in the expansivity by heat treatment are comparatively large, it is always possible that in some of these cases it may be quite advantageous to control the expansivity by modifying the heat treatment. This would be especially true if the glasses in question are of types which are valuable because of their low expansivity. That the expansivity changes obtainable by heat treatment may in some cases materially exceed 3 percent was shown by some preliminary work in which it was found that heat treating a sample of pyrex tubing at $550^{\circ} \mathrm{C}$., which is not an extreme annealing temperature, reduced the expansivity 6 percent or more. ${ }^{2}$ Presumably the tubing had originally been cooled quite rapidly to room temperature after being made; and in that case it doubtless had a relatively high effective treating temperature. This consideration, together with the above result, would make it appear that lowering the annealing temperature of this type of pyrex glass reduces the expansivity materially.

In at least a portion of the annealing range, lowering of the annealing temperature also appears to reduce the specific volume, $V_{s}$, at $20^{\circ} \mathrm{C}$; and, since the expansivity is apparently decreased coincidently, it follows that the corresponding volume change, $\Delta V_{a}$, during the annealing is a greater decrease than the $\Delta V_{s}$ and that the difference between these decrements is greater than that which would be caused by an unchanged expansivity. ${ }^{3}$ It is, however, difficult if

${ }^{2}$ Dimensional Changes Caused in Pyrex Glass by Heat Treatment, informally reported at the 1930 meeting of the American Ceramic Society, Toronto, Canada. The composition of this glass and also that on which the following expansivities were obtained is $\mathrm{SiO}_{2} 80.7, \mathrm{R}_{2} \mathrm{O}_{3} 2.1$, Na2 $\mathrm{O}_{4.0}$, and $\mathrm{B}_{2} \mathrm{O}_{3}$ (by difference) 13.2 percent.

${ }_{3}$ That is, if the original volume of any glass sample at a given temperature, $T_{s}$, is $V_{8}$, and after being heated rapidly to $T_{a}$ (a chosen treating temperature in the annealing range) immediately becomes $V_{a}$, then these volumes may be related by the usual expression, $V_{a}=V_{s}\left[1+\beta\left(T_{a}-T_{s}\right)\right]$, where $\beta$ is the average volume expansivity and has a value determined by the temperature interval, $T_{a}-T_{s}$, and the condition of the glass. During continued heating at $T_{a}$ the volume $V_{a}$ will in time change to a constant value $V_{a^{\prime}}$, and $\beta$ to $\beta^{\prime}$, so that when the sample is ultimately cooled rapidly to $T_{s}$ a volume $V_{s}^{\prime}$ is obtained and

From this it follows that-

$$
V_{a}^{\prime}=V_{s}^{\prime}\left[1+\beta^{\prime}\left(T_{a}-T_{s}\right)\right]
$$

$$
\Delta V_{a}=\Delta V_{s}+\left[\Delta \beta . V_{s}+\beta . \Delta V_{s}\right]\left(T_{a}-T_{s}\right) \text {, }
$$

where $\Delta V_{a}=V_{a}-V_{a}^{\prime}$, etc. If both $V_{s}$ and $\beta$ are decreased after a heat treatment it is evident that these decreases will both contribute in the same sense to a computed value for the change in $V_{a}$. The above equation may also be written in the form

$$
\Delta V_{a}-\Delta V_{s}=\Delta V_{s} \beta\left(T_{a}-T_{s}\right)\left(\frac{\Delta \beta}{\beta} \frac{V_{s}}{\Delta V_{s}}+1\right)
$$

which, assuming that $\beta$ and $T_{a}-T_{s}$ are approximately $3 \times 10^{-5}$ and $500^{\circ}$ respectively for ordinary glasses, makes it obvious that $\frac{\Delta \beta}{\beta}$ must be about nine or ten times as large as $\frac{\Delta V_{s}}{V_{c}}$ if $\Delta V_{a}-\Delta V_{s}$ is to approximate 15 percent of $\Delta V_{s}$.

It should be noted that in developing such relations as the above it may be necessary, in some cases, to include terms containing the square and possibly higher powers of the temperature, especially if the temperature interval considered is large. 
not impossible to measure $\Delta V_{a}$ with an accuracy which would demonstrate this.

If in other portions of the annealing range of this pyrex glass a lowering of the effective treating temperature increases $V_{3}$ as seems to be the case from previous studies, ${ }^{4}$ it follows that a coincident reduction in the expansivity would signify a $\Delta V_{a}$ that is smaller algebraically than $\Delta V_{s}$ and possibly one that indicates a reduction of $V_{a}$ as before. Ordinarily, however, the effect of a change in the expansivity is too small to produce this possible difference in direction between $\Delta V_{s}$ and $\Delta V_{a}$. Moreover, observations on the dimensional changes during the process of annealing pyrex glass will usually show that both changes are ultimately in the same direction although the change in $V_{a}$ may be observed to reverse its trend during the annealing period. ${ }^{5}$ As a matter of fact, this reversal in trend suggests the possibility that at least two processes opposed in their volume effects are developed in this glass by annealing treatments which lower the effective treating temperature, and that the one which increases $V_{s}$ proceeds at a relatively low rate. It is also believed that this possibility will, under certain assumptions, partially explain the minimum densities obtained ${ }^{6}$ by treatments at temperatures near $550^{\circ}$ C. more satisfactorily than any probable change in the expansivity. Before any reliable conclusions could be formed concerning this possibility it seemed necessary that the range of expansivity changes caused by treatments at different annealing temperatures should be studied more thoroughly.

\section{SPECIMENS TESTED AND APPARATUS USED}

The specimens tested were pyrex tubes of the kind ordinarily used in constructing chemical apparatus and had approximately a $12 \mathrm{~mm}$ outside diameter and a $1.5 \mathrm{~mm}$ wall. Ring sections approximately $5 \mathrm{~mm}$ in length were cut from these specimens and ground so that on each end of a section there were three short equally spaced legs with small feet. Before testing such samples, the slightly rounded bearing surfaces of the feet were polished reasonably smooth and so adjusted that the section when acting as a spacer between two flat interferometer plates of pyrex glass ${ }^{7}$ would cause interference fringes of a suitable width to appear when this interferometer system was illuminated in the usual way with monochromatic light (e.g., helium, $\lambda=$ $5875.6 \mathrm{~A})$.

During an expansivity test this interferometer system rested directly on an interferometric thermometer plate of fused quartz in such a way that the fringes formed by this plate as well as those from the interferometer itself could be viewed simultaneously by a previously described $^{8}$ viewing apparatus. This complete system rested in a heavy

4 Tool and Hill, J. Soc. Glass Techn., vol. 9, p. 185, 1925.

5 Linear expansion curves showing this reversal in trend were exhibited in the above mentioned informal report.

${ }^{6}$ Tool and Hill, J. Soc. Glass Techn., vol. 9, p. 185, 1925.

7 It has been found advisable, whenever possible, to use interferometer plates of the same glass as the test specimen. This prevents to a great extent differential expansion and almost eliminates the slippage between the plates and specimen which so often disturbs the equilibrium of the system and causes small but definite breaks and changes in slope in the expansion curves. Such disturbances are particularly apt to occur somewhat above $200^{\circ} \mathrm{C}$. where it appears that the lubricating gas films undergo a considerable change. Pyrex plates can be used to temperatures somewhat above $600^{\circ} \mathrm{C}$., and those of other glasses to temperatures having corresponding positions in the appropriate annealing ranges.

The ring type of specimens used in these tests also adds greatly to the stability of the interferometer system. In some cases still greater stability has been obtained by fusing the spacer and plates into a single unit.

8 Tool, Lloyd, and Merritt, J. Am. Ceramic Soc., vol. 13, p. 653, 1930. 
aluminum cup which was covered by an aluminum lid containing a small, fused-quartz window. During the observations the cup and the contents were heated and cooled in an upright tubular electric furnace at a rate of about $3^{\circ} \mathrm{C}$. per minute. The cooling rate of the furnace could be regulated by forcing air through a chamber which inclosed the heating elements.

\section{HEAT TREATMENTS OF SAMPLES}

Before the final adjustment of the test samples they were divided into lots and each lot was then treated at one of the several chosen treating temperatures spaced at intervals of $25^{\circ}$ from $450^{\circ}$ to $750^{\circ} \mathrm{C}$. A horizontal tubular electric furnace whose temperature could be controlled over a considerable time to within $\pm 5^{\circ} \mathrm{C}$. was employed for such treatments. The periods of treatment employed at these temperatures were based on experience gained during an investigation of the time required to obtain a density equilibrium for this glass at various treating temperatures. (See footnote 4 for reference.) Such periods ranged from 2 months at $450^{\circ}$ to 30 minutes at $750^{\circ} \mathrm{C}$. At $450^{\circ}$ C. a period of 2 months is possibly too short to produce the maximum effect, but it is estimated that it causes in excess of nine tenths of the total change possible. ${ }^{9} \quad$ An appreciably closer approach requires an unduly long treatment. At $750^{\circ} \mathrm{C}$. a period of one half hour may also be somewhat insufficient. The possibility of devitrification, which occasionally becomes noticeable after several hours' treatment somewhat above $650^{\circ} \mathrm{C}$. makes it necessary, however, to reduce the periods as much as possible in this range. The periods at all the higher temperatures were therefore made as short as seemed consistent with a reasonable approach to a stable density while the glass still remained in the undevitrified ${ }^{10}$ state. After treating the glass for the periods mentioned, it was cooled rapidly in air; and, from temperatures above $550^{\circ} \mathrm{C}$., the samples were shaken about on burned asbestos board for the first moments of the cooling. This agitation should make the cooling more uniform and somewhat more rapid. The resultant shortening of the cooling period is especially desirable since there is no doubt that some annealing, which may affect the various possible processes differently, occurs during the time required in cooling to $550^{\circ}$ from temperatures above $600^{\circ} \mathrm{C}$. Cooling at these rates from such temperatures naturally introduces a certain degree of strain and possibly some resultant inconsistency in the observed expansion data, but this cannot be avoided if the effects produced on the expansivity by high temperature treatments are to be preserved for tests at lower temperatures.

\section{METHODS OF OBSERVATION}

As each interference (air) fringe, indicating a change of $1 / 2 \lambda$ or $293.78 \mu$ in the height of the pyrex separator, passed the reference point of the interferometer system they were counted and recorded

9 There were some indications in the previous work on density that very long treatments tended to yield values slightly lower than those reached after shorter ones. Such a result would correspond to the previousincreases the observed avoid if possible the least trace of devitrification since that change in the glass greatly $200^{\circ} \mathrm{C}$ and at the same time makes a determination of the true expansivity impossible. For a discussion of the terms "true" and "apparent" expansivity see Tool and Eichlin, B.S. Jour. Research, vol. 6, p. 523, 1931. 
along with the simultaneously determined number of fringes (including fractions) which had passed the reference point of the refraction thermometer and which indicated the corresponding change in temperature. For the approximate temperature range, $25^{\circ}$ to $200^{\circ} \mathrm{C}$., which was used in these tests the total change in the number of air fringes and thermometer fringes averaged about 9 and 27 , respectively.

Each test consisted of two complete heating and cooling cycles and consequently furnished four determinations of the temperature (as indicated by the accumulated number of refraction thermometer fringes) for each air fringe. The average of these four temperature determinations and the relative change in length in microns per centimeter, $\frac{\Delta l}{L},{ }^{11}$ were used to determine the points for a tentative expansion curve. These points (approximately nine in number) fell on a practically straight line although a large-scale plot usually made noticeable a very slight convexity toward the temperature coordinate.

After a heating and a cooling cycle the measurements usually showed a slight "apparent shortening" of the samples especially in the case of those treated at the higher temperatures. This effect, if it is a real shortening or contraction, is doubtless in some degree similar to such annealing effects as slight deformations under stress and perhaps to very small specific volume changes similar to those found in the normal annealing range although not necessarily of the same sign. It was found, moreover, that these "shortening effects" were usually somewhat larger during the first cycle of heating and cooling; consequently, each sample was subjected to a preliminary cycle before the actual test began. This seemed especially advisable since a large part of the effect, during the first cycle at least, may well be considered as only an apparent contraction caused by an attenuation of the thin air films between the feet of the specimen and the interferometer plates rather than to the above-mentioned possibilities.

Since the tentative expansion curves were all very nearly straight lines and since only the average expansivities for the temperature range were desired, it seemed sufficient to make the assumption that $\left(\frac{\Delta l}{L}\right)_{T}=A+B T$, where $B$ is the expansivity expressed in microns per cm per degree centigrade. To compute $B$ as objectively as possible and, for comparative purposes, as carefully as repeated tests on a given sample appeared to warrant, it seemed desirable to discard the graphical procedure and to use the method of least squares. In the 15 cases where two or more tests were made on a sample, there was only one case in which the total difference between the computed values of $B$ for a given sample was as great as 1 percent. The much larger spreads shown in the table of results arise from the use of more than one sample for the determinations at a given treating temperature.

\section{RESULTS}

The results for $B$ presented in the table and figure are averages of two or more determinations on one or more samples as indicated. While the maximum spread of the results for a given treating tempera-

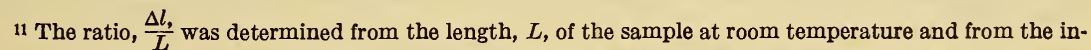
crease in length, $\Delta l$, as computed from the number of air fringes and the wave length of the light. Corrections were employed which allowed for the temperature change in air refractivity.
} 
ture is not in every case as small as might be desired, it is seldom excessive and never sufficient to indicate that the expansivity failed to increase as the treating temperature was increased if the range above $625^{\circ} \mathrm{C}$. is excepted. For treatments in this range, however, relatively erratic results are to be expected as previously noted.

From the results obtained it was found that the linear expansivity, $\alpha\left(=\frac{\beta}{3}\right)$ increases from about $3.075 \times 10^{-6}$ to $3.420 \times 10^{-6}$ as the treating temperature is increased from 450 to $650^{\circ} \mathrm{C}$. This is an increase in excess of 10 percent and agrees well with the tentative value of 6 percent (given in the informal progress report cited above) as the increase for a shorter range. This range probably did not exceed $100^{\circ}$ since, as previously explained, it was limited by the treating temperature for that determination, $550^{\circ} \mathrm{C}$., and the relatively high effective treating temperature of the original glass which probably did not exceed $650^{\circ} \mathrm{C}$.

Some tests made on the original glass during the present investigations give $3.406 \times 10^{-6}$ as the average result for its expansivity. This value would place the original effective annealing temperature of the glass above $625^{\circ} \mathrm{C}$. In this temperature range, however, such results are not definitely indicative of the previous annealing treatment since no great change in the expansivity appears to be produced by treating the glass at temperatures from $625^{\circ}$ to $750^{\circ} \mathrm{C}$. unless incipient devitrification results because of the treatments. This failure of the expansivity to continue to increase with increasing treating temperatures may be interpreted as the result of an annealing ${ }^{12}$ while the glass cools through the range above $600^{\circ} \mathrm{C}$. However, definite proof of this possibility is lacking and is, moreover, difficult to obtain since the temperatures involved cause the glass to become appreciably deformable and subject to the introduction of strains.

After the expansivity has been reduced by a low temperature treatment it can be restored to its previous value by reheating at a higher temperature. This is demonstrated by the result (shown in the table) on a sample retreated at $675^{\circ} \mathrm{C}$. after it had first been treated at $450^{\circ} \mathrm{C}$. with the other samples yielding the results given for the latter temperature. The result on the last sample recorded in the table also shows this reversibility, although the value obtained for the expansivity is not as large as might have been expected for a retreatment

\footnotetext{
12 The higher densities found by Tool and Hill for another specimen of pyrex glass after treatments from $600^{\circ} \mathrm{C}$. up as compared to those found for treatments between $525^{\circ}$ and $575^{\circ} \mathrm{C}$. can to some extent be explained on the basis of the greater expansivities obtained at higher treating temperatures. Nevertheless, there is some indication that the minimum in the density curves may have resulted in part from the annealing received by the samples (which were treated at the higher temperatures) while they cooled through the range above $550^{\circ} \mathrm{C}$. This effect of the annealing would be possible on the basis of the hypothetical processes mentioned in connection with footnote 5 . That is, during cooling, the process causing contraction and occurring before the previously mentioned reversal, progresses much more rapidly and increases the density more than the opposed process acting simultaneously decreases it. This explanation assumes that this latter and slower process is only capable of showing its full relative effect when the annealing temperatures are low enough to prevent (in view of the rapid rate of cooling employed in the tests under consideration) the faster process from making any progress as the glass is cooled after a heat treatment.

The larger samples required for the density tests caused the possible rate of cooling to be lower than in the case of the very small rings used in the present investigation. It is probable, however, that even the more active process ceased to make any great progress soon after either type of sample reached $600^{\circ} \mathrm{C}$. during the cooling involved in their preparation for test.

The composition of the glass used in these density tests is $\mathrm{SiO}_{2} 81.4, \mathrm{R}_{2} \mathrm{O}_{3} 2.2, \mathrm{Na}_{2} \mathrm{O} 4.0$, and $\mathrm{B}_{2} \mathrm{O}_{3}$ (by difference) 12.4 percent. The differences in composition between this glass and that of the tubing previously mentioned are presumably incident to changes in the process of manufacture, since the above glass was produced several years earlier. The chemical analyses of these glasses were made by F. W. Glaze of this Bureau.
} 
as high as $600^{\circ} \mathrm{C}$. This low value is, however, accounted for by the fact that the sample which was first treated at $500^{\circ} \mathrm{C}$. was retreated during an expansion test which extended to $600^{\circ} \mathrm{C}$. and, as required by the test, cooled at the relatively slow rate of $3^{\circ}$ per minute, which again reduced the expansivity somewhat.

Results of tests of this kind indicate the rapidity with which annealing and disannealing proceed. For example, one of the samples previously treated at $450^{\circ} \mathrm{C}$. expanded, when subsequently disannealed by heating at a rate of $3^{\circ}$ per minute and while passing through the $50^{\circ}$ range just above $550^{\circ} \mathrm{C}$., to an amount which was almost equal to the total normal expansion caused by the true expansivity alone in the range from $20^{\circ}$ to $500^{\circ} \mathrm{C}$. Moreover, the expansion developed so rapidly as $600^{\circ}$ was approached that it could not by such a test be distinguished from a normal expansion. In fact, some of this expansion in the range between $550^{\circ}$ and $600^{\circ} \mathrm{C}$. is caused by the true expansivity of the material but it can be shown that at least 50 percent of it can ultimately be obtained by treating at $550^{\circ} \mathrm{C}$. and without going to higher temperatures. Obtaining the expansion in this way shows that a large portion of it is a disannealing effect, although a much longer time is required because the physico-chemical changes involved in disannealing always progress at a greatly decreased rate at lower temperatures. For the same reason it is obvious that the annealing which occurs while cooling at the rate of $3^{\circ}$ per minute from $600^{\circ} \mathrm{C}$. to room temperature will not equal the disannealing caused by the previous heating to $600^{\circ} \mathrm{C}$. at the same rate in such a test as the above; but, if the expansivity results mentioned in the previous paragraph are any criterion, such a cooling did reduce the effective annealing temperature from $600^{\circ} \mathrm{C}$. (the upper limit of the test and the point where the sample was given ample opportunity to reach approximate equilibrium) to $550^{\circ} \mathrm{C}$. The annealing effect caused by such a cooling is, therefore, about one half of the annealing effect obtained when a sample is thoroughly treated at $500^{\circ} \mathrm{C}$., if such effects are measured in terms of effective treating temperature displacements.

\section{GENERAL DISCUSSION}

Even from a practical standpoint small changes in the expansivity such as those found here have some significance in special cases. In glassware which is subjected to sudden temperature changes it is naturally desirable to reduce the expansivity as much as possible and in such cases these small reductions by treatment at relatively low annealing temperatures may be serviceable in reducing breakage from thermal shock. One point of special interest to be noted is that the increase in expansivity caused by treating at high temperaatures will affect to some degree the gain in mechanical strength which may be obtained by so-called "tempering treatments." That is, a higher expansivity should assist somewhat in introducing the required strain to make the "tempered" ware more resistant to mechanical shock, but it may also increase the sensitivity of the ware to severe localized thermal shocks.

$18745-33-6$ 
TABLE 1

\begin{tabular}{|c|c|c|c|c|c|}
\hline $\begin{array}{c}\text { Number } \\
\text { of sam- } \\
\text { ples } \\
\text { tested }\end{array}$ & $\begin{array}{c}\text { Total } \\
\text { number } \\
\text { of tests }\end{array}$ & $\begin{array}{l}\text { Treating } \\
\text { tempera- } \\
\text { ture }\end{array}$ & $\begin{array}{l}\text { Approxi- } \\
\text { mate } \\
\text { treating } \\
\text { time }\end{array}$ & $\begin{array}{c}\text { Average ex- } \\
\text { pansivity } \\
\mathrm{B}^{a}\end{array}$ & $\begin{array}{c}\text { Greatest } \\
\text { spread in } \\
\text { results }\end{array}$ \\
\hline $\begin{array}{l}2 \\
2 \\
2 \\
2 \\
2 \\
1 \\
3 \\
2 \\
2 \\
2 \\
2 \\
2 \\
2 \\
1 \\
1 \\
1\end{array}$ & $\begin{array}{l}3 \\
3 \\
3 \\
3 \\
3 \\
3 \\
4 \\
3 \\
4 \\
3 \\
3 \\
4 \\
3 \\
2 \\
1\end{array}$ & $\begin{array}{l}{ }^{\circ} C . \\
450 \\
475 \\
500 \\
525 \\
550 \\
575 \\
600 \\
625 \\
650 \\
675 \\
700 \\
725 \\
750 \\
(b) \\
c 450 \\
c 675 \\
c 500 \\
c 600\end{array}$ & \begin{tabular}{c} 
Hours \\
1,610 \\
1,750 \\
1,030 \\
720 \\
330 \\
170 \\
120 \\
72 \\
27 \\
5.5 \\
3.0 \\
2.0 \\
.5 \\
\hdashline 1,610 \\
6 \\
1,030 \\
.1
\end{tabular} & $\begin{array}{r}\text { Microns/cm. }{ }^{\circ} \mathrm{C} . \\
0.0307 \\
.0310 \\
.0313 \\
.0317 \\
.0320 \\
.0326 \\
.0329 \\
.0338 \\
.0342 \\
.0342 \\
.0341 \\
.0345 \\
.0343 \\
.0341 \\
.0342 \\
.0320\end{array}$ & $\begin{array}{r}\text { Percent } \\
0.3 \\
.4 \\
1.0 \\
.9 \\
.4 \\
.8 \\
1.0 \\
3.0 \\
4.0 \\
.7 \\
2.0 \\
3.0 \\
1.5 \\
.8\end{array}$ \\
\hline
\end{tabular}

a The values $B$ when multiplied by $10^{-4}$ become the usual linear expansivities or coefficients of linear expansion designated by $a$ in the previous pages.

$b$ Untreated.

c First.

d Second.

- This treatment also included heating and cooling at a $3^{\circ} \mathrm{C}$. per minute rate since it was given during an expansion test extending to $600^{\circ} \mathrm{C}$. The samples in all other cases were cooled as rapidly as possible in air at room temperature.

From the more general viewpoint of gaining an understanding of the behavior of glass under the application of heat treatments such results as those shown in table 1 assume, however, a greater importance. The results on this particular glass show, for example, that the density when determined under standard conditions after various more or less complete heat treatments does not give an entirely adequate idea of the manner in which the density of the glass varies with temperature and time of treatment in the annealing range. They indicate also that the relatively rapid increases in size which are always evident in annealed samples, of this type of pyrex glass when subjected to expansion tests extending above $500^{\circ}$ C. are probably caused to a small degree by an actual increase in the true thermal expansivity as the disannealing progresses. This increase is caused presumably by some of those same physico-chemical processes which produce the changes in volume which require time as well as a change in temperature within the annealing range for their accomplishment. Likewise it also appears probable that the well known slowing up of the rate of apparent expansion between $300^{\circ}$ and $500^{\circ} \mathrm{C}$. shown by expansion curves obtained by heating chilled samples is to some small extent the result of a decrease in the true expansivity as the annealing at lower temperatures progresses.

From the results depicted by the accompanying curves it is evident that the expansivity when determined with sufficient accuracy, possesses an advantage over density measurements as a means of determining the effective annealing temperature of a sample of this particular type of pyrex glass. This follows because it is apparent that in the range between $500^{\circ}$ and $650^{\circ} \mathrm{C}$. two treatments, one above $560^{\circ}$ and the other below, may cause the glass to have the same density (or refractivity), whereas this is not true in the case of the 
expansivity. Another advantage arising from the utilization of expansivity tests for this purpose is found in the possible use of much thinner and lighter samples which can be subjected to a more rapid cooling. In the case of this glass, the rapidity of cooling may be especially important if, as seems possible, there are two types of processes progressing at different rates during heat treatments in the annealing range and producing opposed changes in volume. ${ }^{13}$

In the beginning it was suspected that one of these processes was an adjustment antecedent to the process of devitrification and that it was probably the one which is slower and causes an increase in volume as the treating temperature is lowered. In that case it might also be suspected that its advancement would increase the expansivity in the range between $100^{\circ}$ and $200^{\circ} \mathrm{C}$. since the first product of devitrification so far detected after treatments at temperatures between

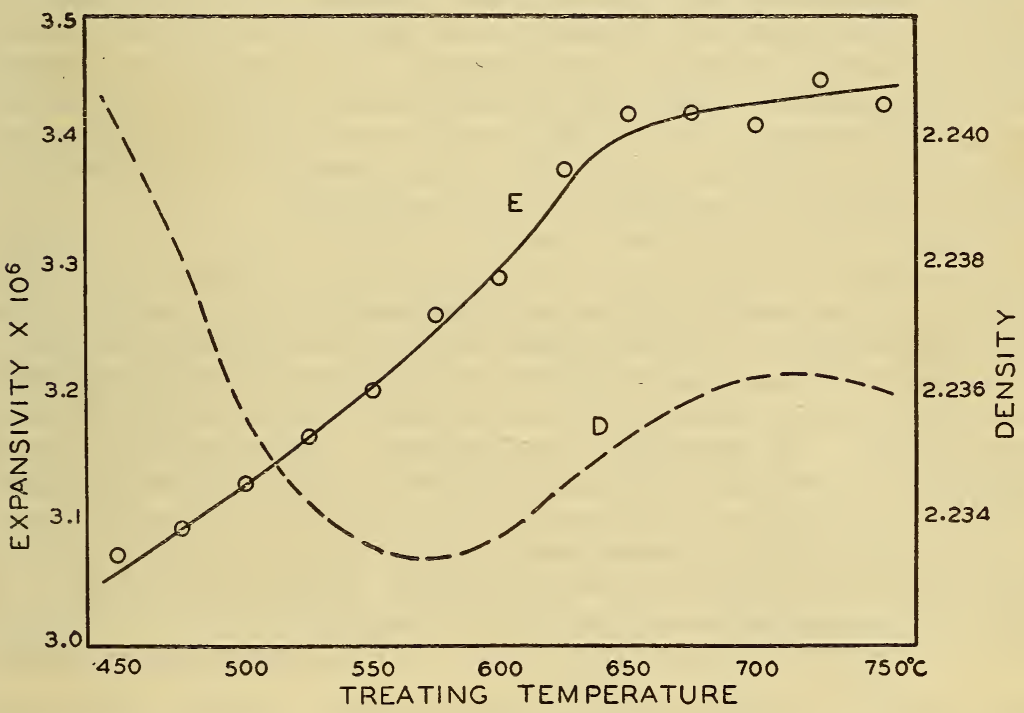

Figure 1.-E. Graphic presentation of data shown in table 1. D. Reproduction of curve oblained by an investigation of the density changes caused by varying the annealing temperature

[See references 2 and 4 . The curve showing the refractivity changes is similar in form.]

$625^{\circ}$ and $750^{\circ} \mathrm{C}$. is that form of cristobalite having an inversion near $200^{\circ} \mathrm{C}$. In any event long treatments designed to advance the slower type of process as far as possible at the various treating temperatures show that something like devitrification is slightly active at points as low as $600^{\circ} \mathrm{C}$.; and even slight traces of visible devitrification in samples so treated appear to cause a definite expansivity increase near the cristobalite inversion range. In the present investigation, however, the treatments employed at temperatures between $550^{\circ}$ and $600^{\circ} \mathrm{C}$. were sufficiently long to advance the volume increases, caused presumably by the slower process, to limits apparently unattainable above $600^{\circ} \mathrm{C}$.; and yet the results show that these treatments reduced the expansivity steadily as the treating temperature was lowered. Moreover, no visible indications of devitrification appeared;

\footnotetext{
${ }^{13}$ See footnote 12 , page 804.
} 
consequently the existence of any direct relation between the volumeincreasing process and that of devitrification seems doubtful.

Below $525^{\circ} \mathrm{C}$. where the volume-decreasing processes are predominant it is also evident that the expansivity is reduced as the volume decrease is enhanced by lowering the treating temperature. This appears to indicate that these processes also are not directly related to devitrification, or at least not when they progress in this temperature range.

Any attempt to determine the idealized relation existing between the specific volume change, $\Delta V_{s}$, which should be obtained at a standard temperature as the result of a heat treatment at some chosen annealing temperature and the corresponding change, $\Delta V_{a}$, which should occur at the annealing temperature during treatment is practically impossible. This is the case because on heating or cooling a sample of the glass through the range between these temperatures and especially through that part above $300^{\circ} \mathrm{C}$. there is always a variable volume change which is not a true thermal expansion or contraction. This change may or may not be a normal portion of the change, $\Delta V_{a}$, which should occur at the annealing temperature but in either case it affects the observed values found for both $\Delta V_{a}$ and $\Delta V_{s}$ and also for the expansivity.

It is of interest to assume, however, that the values for $\Delta V_{s}$ which may be computed from the previously reported densities of a pyrex glass are approximately correct and applicable to the pyrex glass used in this investigation; and also, that the values obtained below $200^{\circ} \mathrm{C}$. for the expansivities of the latter are practically valid for the remainder of the range between this point and the various annealing (or treating) temperatures. On this basis approximate values for $\Delta V_{a}$ may be computed. Thus, the densities at $20^{\circ} \mathrm{C}$. for the previously investigated sample of pyrex glass were 2.2415 and 2.2330 after treatments at $450^{\circ}$ and $550^{\circ} \mathrm{C}$., respectively, while from the above table the expansivities obtained on the supposedly similar type of glass for the same treatments were $0.03074 \times 10^{-4}$ and $0.03204 \times 10^{-4}$. A treatment at $550^{\circ}$ after one at $450^{\circ} \mathrm{C}$. will, therefore, produce the relative increases, $\frac{\Delta V_{s}}{V_{s}}=0.0038$ and $\frac{\Delta \beta}{\beta}=0.041$ in the specific volume and cubical expansivity. On assuming that the relation developed in footnote 3 is representative of the conditions involved, it follows that $\Delta V_{a}=1.06 \Delta V_{s}$ approximately, whereas if the expansivity remained unchanged, the ratio $\frac{\Delta V_{a}}{\Delta V_{s}}$ would not greatly exceed 1.006 for treatments anywhere in the annealing range.

After a treatment at $550^{\circ} \mathrm{C}$. another at $450^{\circ}$ will not produce decreases equivalent to the above increases since it appears that the process is not entirely reversible ${ }^{14}$ between these temperatures. Between $450^{\circ}$ and $675^{\circ} \mathrm{C}$, , however, practically complete reversibility appears to exist. For treatments at these temperatures the densities obtained were 2.2415 and 2.2365 , respectively, while the expansivities shown in the table are $0.03074 \times 10^{-4}$ and $0.03423 \times 10^{-4}$. From these values ${ }^{15}$ it follows that $\Delta V_{a}=1.33 \Delta V_{s}$ which would indicate that

\footnotetext{
14 Numerous ranges can be chosen where there is likely to be this lack of reversibility if the upper and lower limits respectively lie in the ranges $560^{\circ}$ to $650^{\circ}$ and $450^{\circ}$ to $560^{\circ} \mathrm{C}$. Moreover, ranges can be chosen where $\Delta V_{a}$ is practically zero and under such conditions $\Delta V_{a}$ becomes approximately $\Delta \beta . V_{a}\left(\mathrm{~T}_{a}-T_{s}\right)$.

15 In these computations it has generally been assumed that $T_{a}$ was the upper treating temperature.

Obviously, using the lower temperature as $T_{a}$ would alter the result somewhat.
} 
in this case the relative magnitude of the two increments was appreciably affected by the expansivity change. Moreover, between $550^{\circ}$ and $675^{\circ} \mathrm{C}$., which is also a range of apparently complete reversibility, it will be found from the data given above that $\Delta V_{a}=0.72$ $\Delta V_{s}$. In this case $\Delta V_{a}$ is the smaller change and both are decrements when the final treatment is at the higher temperature. To check this result by an experimental determination of $\Delta V_{a}$ is, however, especially difficult since at $675^{\circ} \mathrm{C}$. the glass is somewhat deformable.

This comparatively large effect of the expansivity change in the two latter cases is due to the relative smallness of $\Delta V_{s}$ and $\Delta V_{a}$ for this range of treating temperatures, and in this connection attention must again be directed to the possibility that the negative increment in $V_{s}$ for treating temperatures from $550^{\circ}$ to $675^{\circ} \mathrm{C}$. is to some degree the result of an annealing during the cooling from $675^{\circ}$. If this selective annealing effect between different processes actually exists, it should be reduced to some extent by the use of much smaller samples. For the present it seemed unnecessary, however, to investigate this possibility, expecially in view of the fact that the samples used in both the expansivity and density tests had the minimum size consistent with good results and were also small enough to allow a cooling rate much greater than that which would normally be employed in working the glass.

In conclusion it must be made clear that the expansivity results presented here are intended only as an indication of the range of variation possible as a result of altering the heat treatment of this particular glass. That is, these results must not be considered as typical of all pyrex glass, because in addition to the changes produced by heat treatment it is evident that differences in expansivity are caused by variations in composition between samples and possibly by other factors. Moreover, other possible combinations of heat treatments which may increase and complicate the expansivity changes nave not been attempted in these tests. From the results presented it appears, however, that the points mentioned in the following summary have been established.

\section{SUMMARY}

1. Lowering the annealing temperature in the range $650^{\circ}$ to $450^{\circ} \mathrm{C}$. reduced the mean expansivity of a pyrex glass materially in the range $20^{\circ}$ to $200^{\circ} \mathrm{C}$.

2. The original expansivity of the tubing corresponded to that obtained by treating the glass at temperatures near $650^{\circ} \mathrm{C}$.

3. After the expansivity of an untreated specimen of this glass had been reduced by annealing at relatively low temperatures, it was restored to its former value by retreating at temperatures near $650^{\circ} \mathrm{C}$.

4. Except for certain narrow temperature intervals, a shift in the effective annealing temperature within the annealing range produced, during an annealing or disannealing, a volume change whose absolute magnitude usually exceeded quite materially that of the volume change developed between room temperature and the annealing range as the result of a change produced coincidently in the true expansivity by such a shift. The volume changes observed at a standard room temperature and caused by shifts in the effective 
annealing temperature may be either the sum or difference of these two types of volume change (absolute values).

5. A sample of this glass having the maximum density so far attained by a treatment at $450^{\circ} \mathrm{C}$. will on being retreated at $550^{\circ}$ increase in volume during the annealing by an amount which approaches 50 percent or more of the cubical expansion during the heating from $20^{\circ}$ to $500^{\circ} \mathrm{C}$; ; consequently, a slow rate of heating in the range $450^{\circ}$ to $550^{\circ} \mathrm{C}$. results in an average apparent thermal expansivity greatly exceeding the true value of the expansivity of the glass in this range.

6. Extreme changes in annealing procedure may produce a change as great as 10 percent in the expansivity, and this, together with the accompanying volume change, may be of some practical significance in certain cases.

7. These changes do not appear to be directly related to the process of devitrification.

Washington, D.C., May 17, 1933. 